\section{Focus on Membrane dynamics}

\section{Cellular membranes in eukaryotes are dynamic structures; this is a key property for their roles in numerous cellular processes. In this issue, we present a series of review articles that highlight recent developments in membrane trafficking, and provide an overview of the importance of trafficking events in development and disease.}

Proteins and lipids follow prescribed trafficking pathways in the cell. Nascent proteins shuttle from the endoplasmic reticulum (ER) to the Golgi and onwards to their ultimate cellular destinations, whereas plasmamembrane proteins are internalized and then degraded or recycled back to the membrane. Clathrin-mediated endocytosis is a major mechanism by which cell-surface proteins are internalized, and many fundamental advances in understanding this key trafficking pathway were made in yeast. Sandra Lemmon and colleagues review the process of clathrinmediated endocytosis in yeast, and discuss similarities and differences between this process in yeast and mammalian cells.

Many transport carriers are enrobed by coat proteins, which have been ascribed diverse roles from membrane bending or stabilization of deformed membranes to cargo and adaptor recruitment. In the yeast secretory pathway, transport between the ER and Golgi is mediated by COPII-coated vesicles, and conserved COPII machinery also supports trafficking from the ER in mammalian systems. Randy Schekman and colleagues discuss recent advances in COPII-mediated transport in mammals, including the process of COPII carrier formation and the regulation and function of ER exit sites, where these carriers are generated.

The actin cytoskeleton regulates membrane dynamics to deform the membrane, promoting invagination, tubulation and scission of transport carriers in the secretory and endocytic pathways. Cytoskeletal tracks then support subsequent motor-based transport of these carriers. Mihaela Anitei and Bernard Hoflack discuss how the cytoskeleton and trafficking machinery coordinately regulate transport carrier biogenesis and transport.

Peter Cullen and Hendrik Korswagen outline the many roles of sorting nexins in the endocytic network. Sorting nexins are key components of the retromer complex that rescues internalized proteins from lysosomal degradation by diverting them to the retrograde endosome-to-Golgi trafficking pathway. As part of the retromer complex, the multifaceted sorting nexin proteins have been suggested to bend membranes and recruit cargo, supporting their key role in several developmental processes.

The ESCRT machinery regulates the biogenesis of multivesicular bodies, intermediate compartments in the endocytic pathway that facilitate sorting of proteins destined for degradation by lysosomal proteolysis. Emerging evidence also points to an important role for the ESCRT complexes in development. Harald Stenmark and colleagues review recent data that indicate the importance of ESCRTs in regulating polarity, autophagy and other cellular processes.
This Focus issue on membrane dynamics offers a unique perspective on the cellular apparatus involved in the secretory and endocytic pathways, and provides a current view of the intersection between membrane dynamics and other fundamental cellular processes. An accompanying online library presenting selected research papers and reviews from Nature Cell Biology and other Nature journals can be found on the Focus on Membrane dynamics homepage. We are pleased to bring this series of review articles to our readers, and thank our authors and reviewers for their contributions.

\section{Limited stay for foreign scientists in the UK?}

\section{New immigration proposals restricting the period of stay for highly skilled migrants in the UK could undermine the future of British science.}

The UK government's new proposals to severely limit the path to settlement or permanent residency for skilled migrants from nonEU countries have renewed concerns about the impact of current immigration policies on British science. Details outlined in a Home Office consultation document suggest that the majority of skilled migrants (also known as 'Tier 2 migrants') and their dependents would be expected to leave the country after a maximum stay of five years. The consultation document outlines opportunities that may arise for Tier 2 migrants to switch to a path that would lead to settlement in the UK, including, for example, an automatic route that is at present available for "ministers of religion, elite sportspeople and those earning over $£ 150,000$ ”. However, the vast majority of scientists, including senior investigators, will fail to meet the current salary criterion. For senior researchers, the prospect of uprooting a research programme in five years, often the length of time taken to simply get a programme underway, is likely to be a powerful deterrent to accepting positions in the UK. Similarly, restrictions on the length of stay could force postdoctoral researchers to leave the country before wrapping up a project. A letter organized by CaSE (Campaign for Science \& Engineering in the UK), signed by twenty influential representatives drawn from science and technology, makes these and other crucial points.

New immigration policies imposing annual caps on the entry of skilled migrants, finalized earlier this year, prioritized applicants to "shortage occupations", including several that are science related. In addition, the government announced this summer that the Royal Society could nominate up to 300 scientists of "exceptional talent" to enter the UK. Any gains from these marginally more scientist-friendly immigration policies could be quickly wiped out with the passage of these new measures.

Science is a global enterprise. Draconian measures that would make the UK a less attractive destination for the world's brightest cannot serve to secure the future of British science. Now is the time for concerned scientists to reach out to their elected representatives. 